\title{
Laser Ablation and Laser Direct Writing as Enabling Technologies for the Definition of Micro-Optical Elements
}

\author{
Nina Hendrickx ${ }^{* 1}$, Himanshu Suyal ${ }^{2}$, Geert Van Steenberge ${ }^{1}$, Aongus McCarthy ${ }^{2}$, Andy Walker ${ }^{2}$, \\ Heidi Ottevaere ${ }^{3}$, Hugo Thienpont ${ }^{3}$, Mohammed Taghizadeh ${ }^{2}$, Peter Van Daele ${ }^{1}$ \\ ${ }^{1}$ Ghent University (UGent), TFCG Microsystems, Dept. Information Technology (INTEC), Ghent, \\ Belgium \\ ${ }^{2}$ Heriot-Watt University (HWU), School of Engineering and Physical Sciences, Physics, \\ Edinburgh, Scotland \\ ${ }^{3}$ Vrije Universiteit Brussel (VUB), Dept. of Applied Physics and Photonics (TONA), Brussels, \\ Belgium
}

\begin{abstract}
A qualitative comparison is made between laser direct writing and laser ablation as enabling technologies for the structuring of multimode waveguides $\left(50 \times 50 \mu \mathrm{m}^{2}\right)$ and $45^{\circ}$ micro-mirrors into an optical layer. A small demonstrator is fabricated that allows us to couple light vertically from a transmitter into an optical layer and from the optical layer to a receiver. The optical layer, a multifunctional acrylate-based photo-polymer, is applied on an FR4-substrate. Multimode waveguides, that carry signals in the plane of the optical layer, are fabricated by means of laser direct writing, a technology that is available at HWU. The $45^{\circ}$ micro-mirrors, that provide out-of-plane coupling, are ablated with the laser ablation set-up available at UGent. This set-up contains a KrF-excimer laser $(248 \mathrm{~nm})$ that can be tilted, which eases the definition of angled facets. Surface roughness measurements are performed on both the optical layer and the micro-mirrors with a non-contact optical profiler. Loss measurements are performed on both the waveguides and the micro-mirrors.
\end{abstract}

Keywords: Coupling structures, laser direct writing, laser ablation, optical waveguides, optical interconnects

\section{INTRODUCTION}

It is predicted that the increase in microprocessor clock rate will create bandwidth limitations for copper interconnects on printed circuit boards (PCBs) due to signal attenuation, EMI/EMC problems, and crosstalk ${ }^{1}$. Optical interconnections may overcome some of the bandwidth limitation of electrical interconnects on conventional PCBs. In long haul high-speed communication systems, fiber optic links dominate over copper wires due to the superior bandwidth-distance performance of the optical channel. However, as distances become shorter, copper-based interconnects become the dominant solution because they are simpler, cheaper and reliable. Since printed circuit boards will continue to be among the most important components of electronic equipment, compatibility with the standard PCB manufacturing processes is required in order to be able to create a cost-effective solution. As it is not necessary to design all interconnects for high bandwidth transmission, the present electrical interconnection technology will remain sufficient for transporting the majority of signals. Optical interconnects will only be implemented for connections where a high bandwidth is essential for the performance of the system. For this reason, and from an economical point of view, priority should be given to further development of the existing technology rather than developing a completely new one.

The integration of optical interconnections on a board-level, covering distances from a few $\mathrm{cm}$ to a couple of $\mathrm{m}$, can be done in a variety of ways. One possible approach that has been studied thoroughly in the past is the integration of optical fibers on a $\mathrm{PCB}^{2}$. Optical backplanes based on this solution are commercially available, but are quite expensive and expendable. Another approach that is studied by groups worldwide is the integration of an optical layer in or on a

* nina.hendrickx@intec.ugent.be; phone +32 9 2645370; fax +32 92645374

Integrated Optics: Theory and Applications, edited by Tadeusz Pustelny, Paul V. Lambeck, Christophe Gorecki, Proc. of SPIE Vol. 5956, 59561B, (2005) · 0277-786X/05/\$15 - doi: 10.1117/12.622904 
PCB. In this way, an optical PCB is created which is in fact a hybrid optical-electrical board. The optical layer, that contains optical waveguides and possibly also other passive optical structures, may be an alternative for the transmission of high data-rate, high-density signals.

The structuring of the optical layer can be done in a variety of ways: photolithography ${ }^{3}$, embossing ${ }^{4}$, laser direct writing ${ }^{5}$, laser ablation ${ }^{6}$. We will use laser direct writing and laser ablation for the definition of multimode waveguides and $45^{\circ}$ micro-mirrors into the optical layer. Both technologies are flexible and allow for rapid prototyping. HWU and UGent build a joint demonstrator that provides vertical in- and out-coupling. The demonstrator contains laser direct written waveguides (HWU) and laser ablated out-of-plane turning mirrors (UGent). In sections 2 and 3, a short description is given of the technologies used for the structuring of the optical layer. Section 4, more details are given on the fabrication of the demonstrator board. In section 5, the results from the measurements on the waveguides and the micro-mirrors are discussed.

\section{LASER DIRECT WRITING}

The system, illustrated in Fig. 1(a), is based on a He-Cd $(325 \mathrm{~nm})$ UV-laser and a vertically mounted slotted base-plate which is used to align the polarizing, routing and focusing optics that guide and focus three beams (at normal and $\pm 45^{\circ}$ angles) onto a computer controlled XY translation stage. A flat-top intensity profile for the direct writing of the polymer is achieved by placing a $\sim 60 \mu \mathrm{m}$ square aperture on the axis of the $\sim 1.0 \mathrm{~mm}$ diameter $\mathrm{TEM}_{00}$ Gaussian beam and imaging it onto the polymer using two $80 \mathrm{~mm}$ focal length lenses. The size of the aperture chosen depends on the size of the features required and the shrinkage in the polymer as it cures.

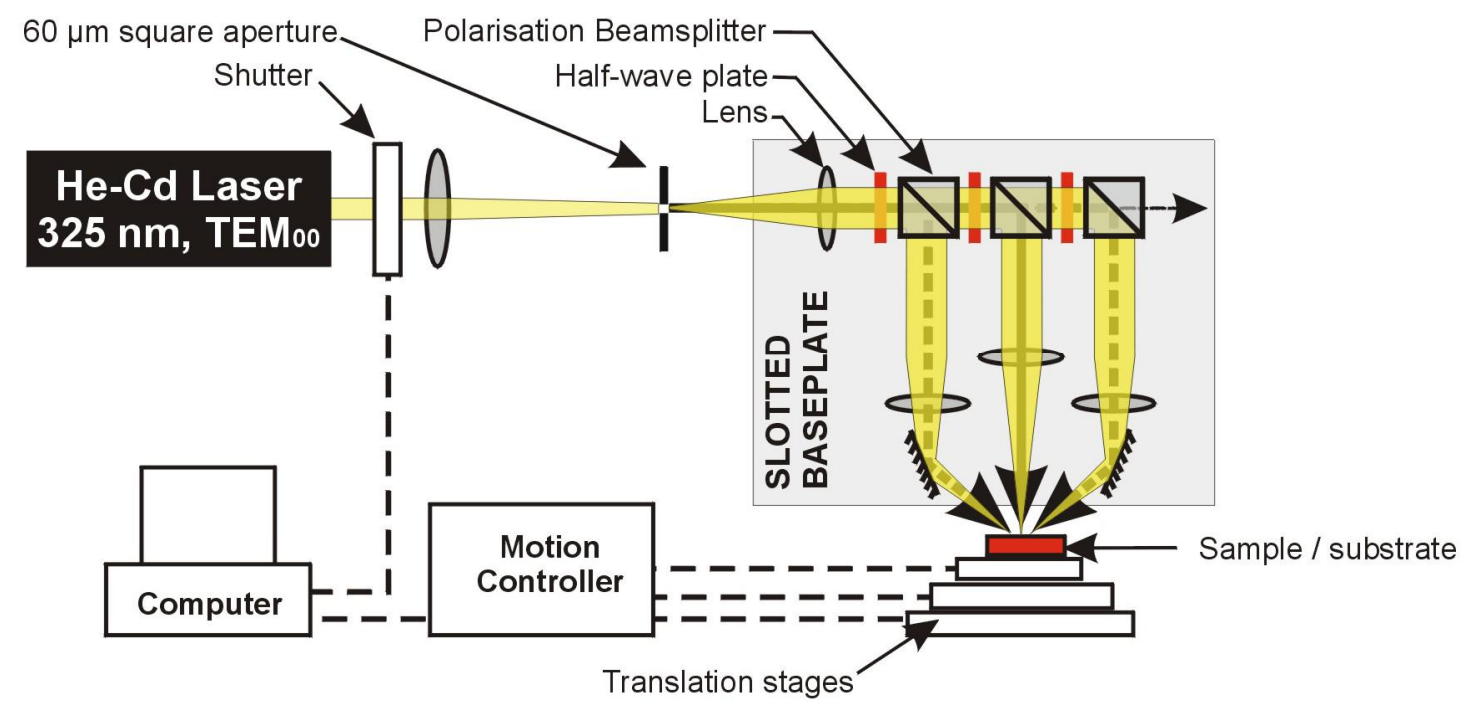

Figure 1: A schematic of the system used for the laser direct-writing of a custom photo-polymer: an aperture imaging setup is used to obtain a flat-top intensity profile for the writing of the polymer.

\section{LASER ABLATION}

Laser ablation is a flexible technology that can be used for the structuring of a large variety of materials. The technique is compatible with standard PCB manufacturing, and is already used for the laser drilling of micro-vias in high density boards. It is a non-mask based technology that can be used for rapid prototyping, as opposed to mask-based approaches where a mask first has to be designed and produced before the optical layer can be patterned. The ablation set-up, shown in Fig. 2, available at UGent contains three different laser sources: a KrF excimer (248nm), a frequency tripled Nd-YAG 
$(355 \mathrm{~nm})$ and a $\mathrm{CO}_{2}(9.6 \mu \mathrm{m})$ laser. Because of the availability of the different laser sources, a large variety of materials can be structured such as polymers, ceramics and glass. The excimer laser can be tilted, which eases the definition of angled facets considerably. The sample is during the processing placed on a computer-controlled translation stage, which has an accuracy of $1 \mu \mathrm{m}$. The three laser sources are available on the same set-up; as a consequence, the sample can stay on the same stage even when different sources are used for the patterning of the material. This approach allows us to achieve high alignment accuracies.

The ablation set-up can be used for the definition of micro-optical elements such as optical waveguides, deflecting optics, refractive lenses and alignment features. This allows for the definition of a complete optical interconnection by means of only one technology ${ }^{6}$. One of the main advantages of ablation with respect to other technologies is the fact that micro-optical elements can be defined on the surface of a heterogeneous opto-electronic module in a very late stage of processing. Ablation is particularly well suited for the structuring of polymer materials because of their excellent UVabsorption behavior and highly non-thermal ablation behavior.
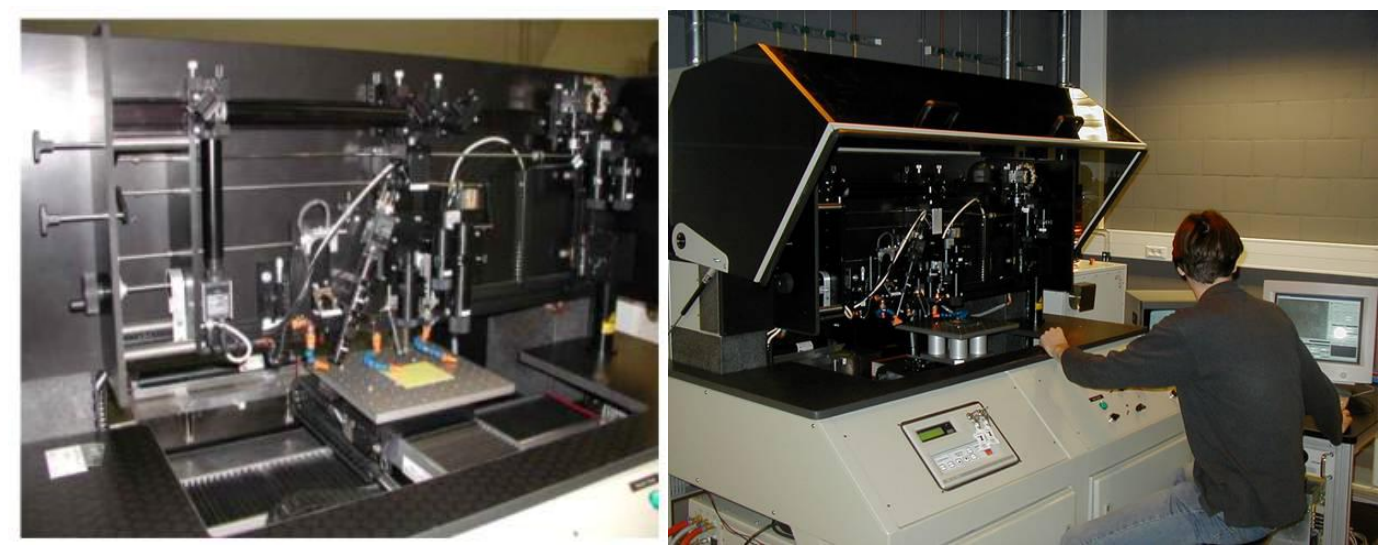

Figure 2: laser ablation set-up available at UGent.

In order to achieve ablation, a laser beam is focused on a sample by means of an optical projection system. The photon energy is absorbed in a thin layer of material. As soon as the photon density inside the material exceeds a certain threshold, the photon energy can be used for material decomposition. The decomposition of material occurs through photo-thermal degradation and photo-chemical decomposition. One of these two processes will be dominant, depending on the nature of the laser pulse and the material that is ablated. In case the photon energy is efficiently absorbed in the material, the photo-chemical process will dominate allowing a clean or "cold" ablation. The decomposed material is ejected, forming a plume of ablation products. Non-evaporated particles in this plume will drop back on or close to the ablated area. The deposition of these particles is called debris and is undesired since it increases the surface roughness of the ablated area. The ablation process is schematically presented in Fig. 3. 


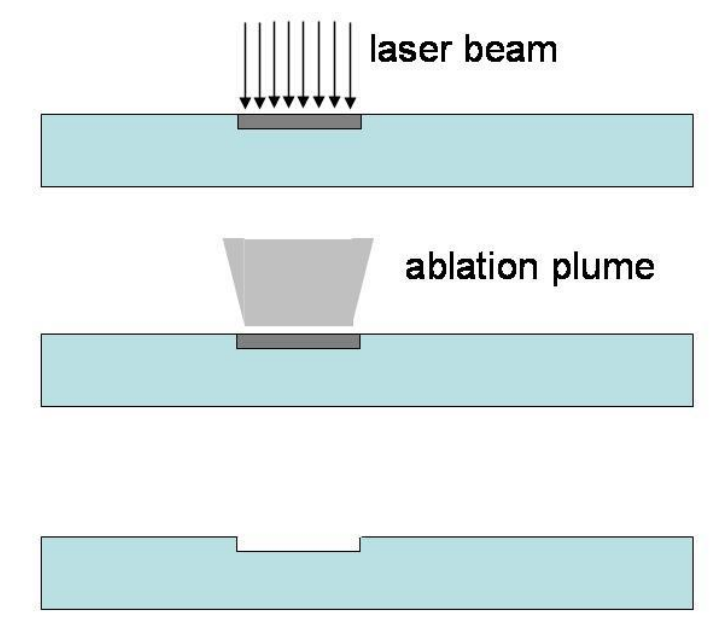

Figure 3: principle of the laser ablation process.

\section{DEMONSTRATOR}

\subsection{Description of the demonstrator}

The demonstrator board presented in this paper is fabricated in the framework of NEMO (network of excellence in micro-optics). It consists of an FR4 substrate on which an optical layer is applied. The optical layer contains an undercladding, core and uppercladding layer. The core layer is patterned by means of laser direct writing, and contains three waveguide arrays with each five waveguides. $45^{\circ}$ micro-mirrors are laser ablated in the optical layer to provide out-of-plane coupling. This allows us to couple light vertically from a transmitter into the plane of the waveguides and from the plane of the waveguides towards a receiver. The demonstrator allows us to investigate the compatibility of laser direct writing and laser ablation and also to compare components fabricated with both technologies.

\subsubsection{Application of the optical layer}

A custom-made, multifunctional acrylate photo-polymer is being used for this work. This novel polymer material has been used to make cladded multimode waveguides and angled structures for out-of plane turning mirrors. The thermal properties of the polymer (e.g. $\mathrm{T}_{\mathrm{g}} \sim 200{ }^{\circ} \mathrm{C}$ ) makes it compatible with the flip-chip bonding process. It has low optical losses at $850 \mathrm{~nm}$, and the refractive index and viscosity can be tuned to suit the process requirements. The polymer has good mechanical properties and good adhesion to substrates such as glass, $\mathrm{SiO}_{2}, \mathrm{Si}$, polystyrene and FR4. Further details of the polymer can be found in the reference ${ }^{5}$.

The polymer behaves like a negative photo-resist in that the areas which are exposed to UV become cured and rest of the uncured polymer is washed away in the development process. The liquid photo-polymer is applied to the substrate using spinning, casting, flow coating or doctor blading. Spinning is used to apply the $50 \mu \mathrm{m}$ thick films of polymer in which features such as waveguide cores and angled surfaces are written. The casting technique is used to deposit the cladding layers and to planarize substrates that contain polymer features. Flow coating and doctor blading are used to apply thicker layers. Large areas of the liquid polymer, such as cladding layers, are cured by placing the sample under a UV lamp in a nitrogen atmosphere. The writing of discrete features such as waveguide cores is achieved using the laser direct-writing system. 


\subsubsection{Laser direct written optical waveguides}

The multimode waveguides are formed by the laser writing of a spun on liquid photo polymer layer. A $50 \mu \mathrm{m}$ thick core layer $(\mathrm{n}=1.579)$ is applied on top of a cured lower cladding layer. The $\mathrm{HeCd}(325 \mathrm{~nm})$ laser beam is send through a $60 \mu \mathrm{m}$ square aperture and imaged on the polymer material. The sample is placed on the computer-controlled XY translation stage and moved with respect to the laser beam. This allows a trajectory of material to be exposed by the UV light as the computer-controlled translation stage moves the substrate with a controlled velocity. The relation between the exposure time and the polymer composition has been carefully characterized. After the exposure the unpolymerized monomer solution is washed off using standard solvents. The patterned core layer is overcoated with a layer of cladding material, which has a refractive index of 1.566. In Fig. 4, some pictures are shown of the laser direct written waveguides. The first to pictures show a cross-section of a waveguide (cross-section $50 \mu \mathrm{m} \times 50 \mu \mathrm{m}$ ), the last picture shows a SEM image of the top view of an array of waveguides at a varying pitch.
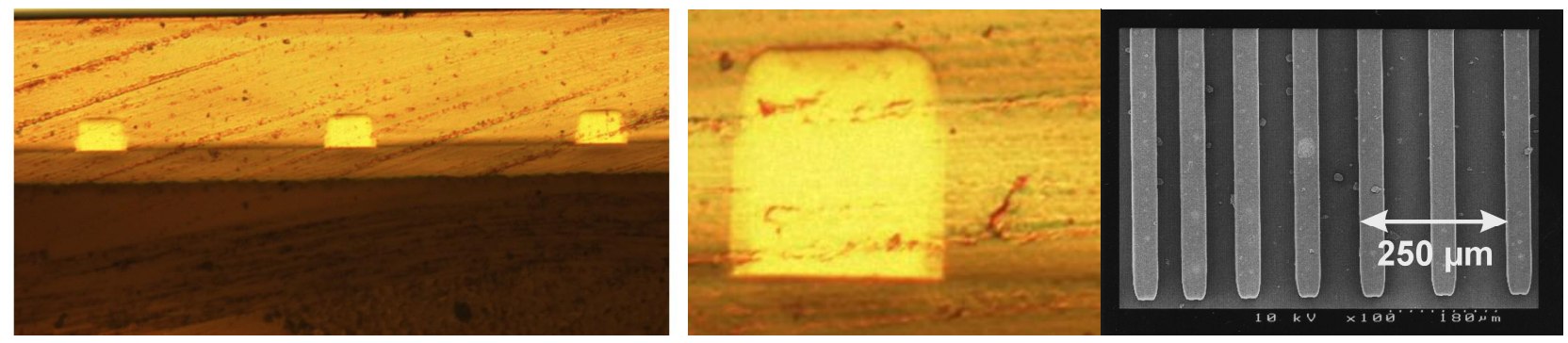

Figure 4: the left picture shows a cross-section of a set of laser direct written waveguides, the middle picture shows a close-up view of a waveguide, the right picture shows a SEM image of the top view of an array of waveguides at a varying pitch.

The final demonstrator board contains three arrays of each five laser written waveguides with a length of $15 \mathrm{~mm}$ on a pitch of $250 \mu \mathrm{m}$. The waveguide cores are written with an optical power of $50 \mu \mathrm{W}$ at a velocity of $60 \mu \mathrm{m} / \mathrm{s}$.

\subsubsection{Laser ablated $45^{\circ}$ micro-mirrors}

The waveguides that are defined in the optical layer allow us to transport signals in the plane of the waveguides. They carry a signal from a transmitter, for instance a VCSEL, towards a receiver, for instance a photodiode or an optical fiber. In order be able to couple the signal from the transmitter towards the waveguide and from the waveguide towards the receiver, the light beam has to be deflected over an angle of $90^{\circ}$. For this purpose, $45^{\circ}$ micro-mirrors are defined in the optical layer to provide out-of-plane coupling. These $45^{\circ}$ micro-mirrors are based on the total internal reflection that occurs at the polymer-air interface. The principle is shown in Fig. 5.

The micro-mirrors are ablated with the KrF excimer laser $(248 \mathrm{~nm})$ that can be tilted; the sample can thus be placed on the translation stage horizontally during processing. The tilt angle can be manually set to the desired value. The laser beam is send through a rectangular aperture and is projected on the sample with an optical projection system. The micro-mirrors are ablated dynamically, meaning that the translation stage is moved with respect to the laser beam during the ablation. There is always a certain degree of tapering; the amount of tapering depends on the material that is being ablated and the laser source that is being used. The tapering angle can be measured experimentally and corrections can be made by adjusting the tilt angle in the appropriate way. As a consequence, either the negative or the positive facet will show an angle of $45^{\circ}$.

Prior to the definition of the micro-mirrors, both the ablation behavior of the material and the tapering angle has to be determined. The tilt angle that allows for the ablation of both a negative and a positive facet under an angle of $45^{\circ}$ is determined. As soon as these properties are known, the $45^{\circ}$ micro-mirrors can be ablated with varying operating parameters (pulse energy, frequency, ablating speed). In Fig. 6, a cross-section and SEM picture is shown of a $45^{\circ}$ negative facet.

The waveguide arrays that are defined in the optical layer are not visible with the camera that is available on the ablation set-up. In order to align the micro-mirrors with respect to the waveguide arrays, three alignment marks are placed on the sample. The alignment marks are deposited by evaporating metal on the lower cladding layer and a lift-off process. On 
the final demonstrator board, the micro-mirrors are ablated dynamically. The frequency and ablation speed are chosen differently for each waveguide array; this allows us to get an idea of the influence of these parameters on the coupling loss of the micro-mirrors.
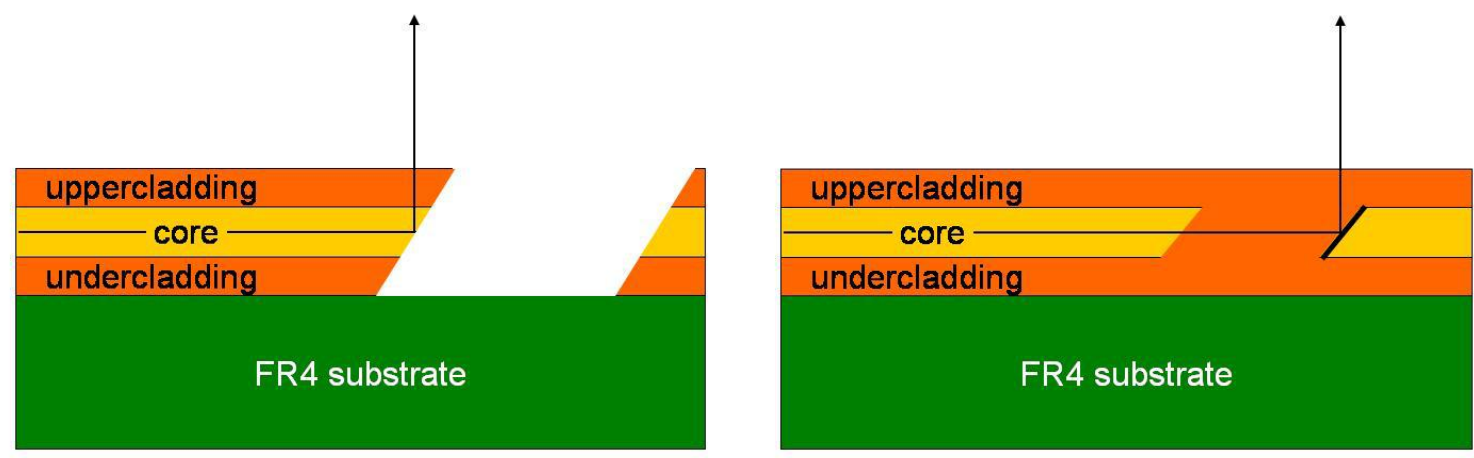

Figure 5: out-of-plane coupling: the left picture shows the principle of the use of a $45^{\circ}$ negative facet, the left picture of the use of a $45^{\circ}$ positive facet.

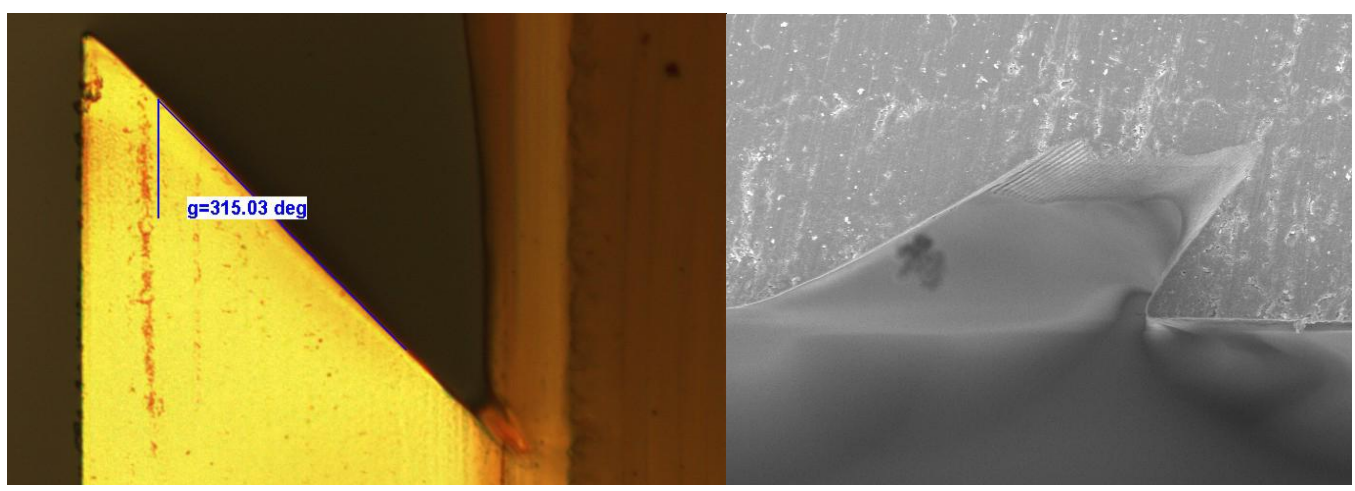

Figure 6: the left picture shows a cross-section of an ablated $45^{\circ}$ negative facet, in the right picture an SEM image is shown of a $45^{\circ}$ negative facet.

\section{MEASUREMENTS}

\subsection{Surface roughness measurements}

Surface roughness measurements are performed on both the optical layer and the ablated facets. The measurements are performed with a non-contact optical profiler available at Free University Brussels (VUB), Brussels.

\subsubsection{Optical layer}

The FR4 substrate has a relatively rough surface. In order to obtain waveguides in the optical layer with a sufficiently low propagation loss the material has to possess planarization properties. The optical layer has an RMS surface roughness of $261 \mathrm{~nm}$ on a scan length of $2300 \mu \mathrm{m}$ and an RMS surface roughness of $65 \mathrm{~nm}$ on a scan length of $1200 \mu \mathrm{m}$. The results are represented in Fig. 7 and demonstrate the planarization properties of the used polymer material. 

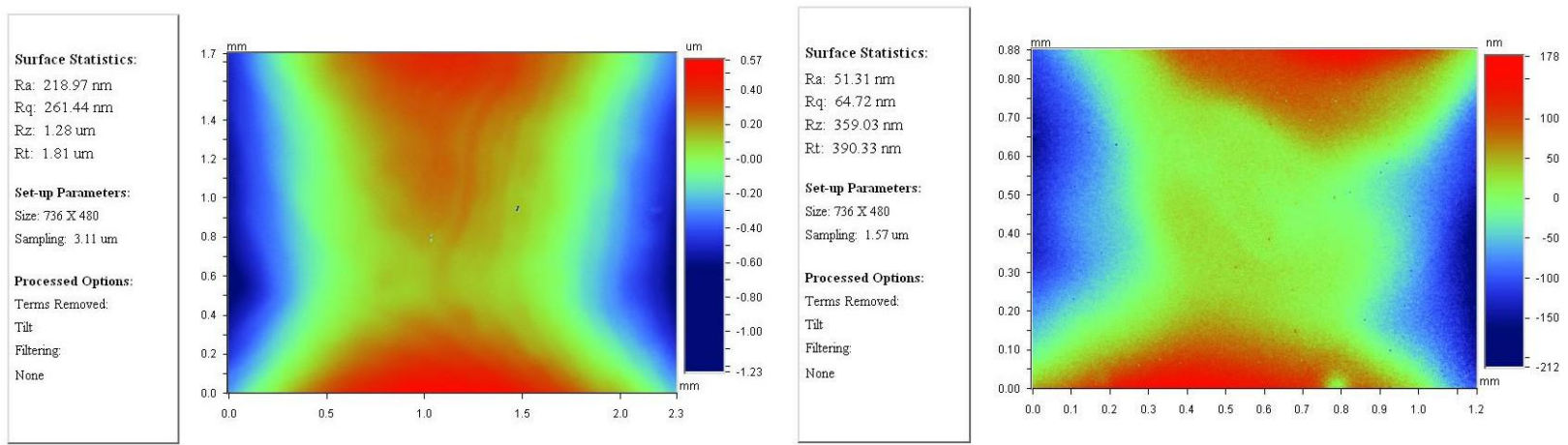

Figure 7: the left picture shows the surface roughness of the optical layer on a scan length of $2300 \mu \mathrm{m}$, the right picture shows the surface roughness on a scan length of $1200 \mu \mathrm{m}$.

\subsubsection{Laser ablated micro-mirrors}

The surface roughness of the ablated facets depends quite strongly on the operating parameters of the laser: frequency, pulse energy and ablation speed. Different combinations of operating parameters have been experimentally tested. In Fig.8, the results are shown measured on a micro-mirror ablated with following parameters:

- Pulse energy: $6 \mathrm{~mJ}$

- Frequency: $200 \mathrm{~Hz}$

- Ablation speed: $248 \mu \mathrm{m} / \mathrm{s}$

The RMS surface roughness of the ablated facet is $186 \mathrm{~nm}$ on a $293 \mu \mathrm{m}$ scan length. This value is relatively high; work is in progress in order to decrease the surface roughness of the ablated facets.

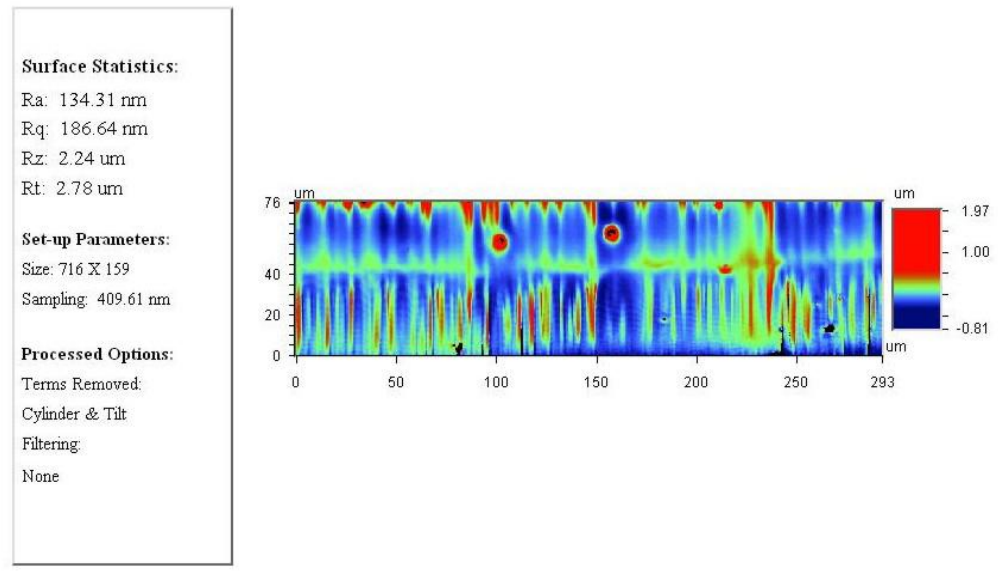

Figure 8: surface roughness measurements on an ablated $45^{\circ}$ facet.

\subsection{Loss measurements}

The characterization set-up that is used to do the measurements is presented in Fig. 9. The optical performance of the waveguides and the mirrors is measured at $850 \mathrm{~nm}$. The propagation loss of the waveguides is determined by a cut-back technique to be $\sim 0.8 \mathrm{~dB} / \mathrm{cm}$. The mirror losses are obtained by comparing the output power of a straight waveguide and a straight waveguide terminated with an out-of-plane turning mirror on one side. The mirror losses are measured to be 
$\sim 1.5 \mathrm{~dB}$. In Fig. 10, some pictures are shown of light coupled into the waveguide horizontally, deflected over $90^{\circ}$ at the mirror and coupled out vertically.

The performance of the mirrors can be improved by decreasing the surface roughness of the negative facet. Work on this subject is in progress. As an alternative, the use of the positive facet can be considered. First tests point out that the surface roughness of the positive facet is lower than the one of the negative facet. In order to be able to use the positive facet for out-of-plane coupling, a thin layer of metal is deposited on the positive facet. The principle is shown in Fig. 5. The $45^{\circ}$ positive facet is ablated in the core layer. After the ablation, a thin layer of metal is deposited. The air gap between the positive and negative facet is filled with cladding material when the upper cladding layer is applied.

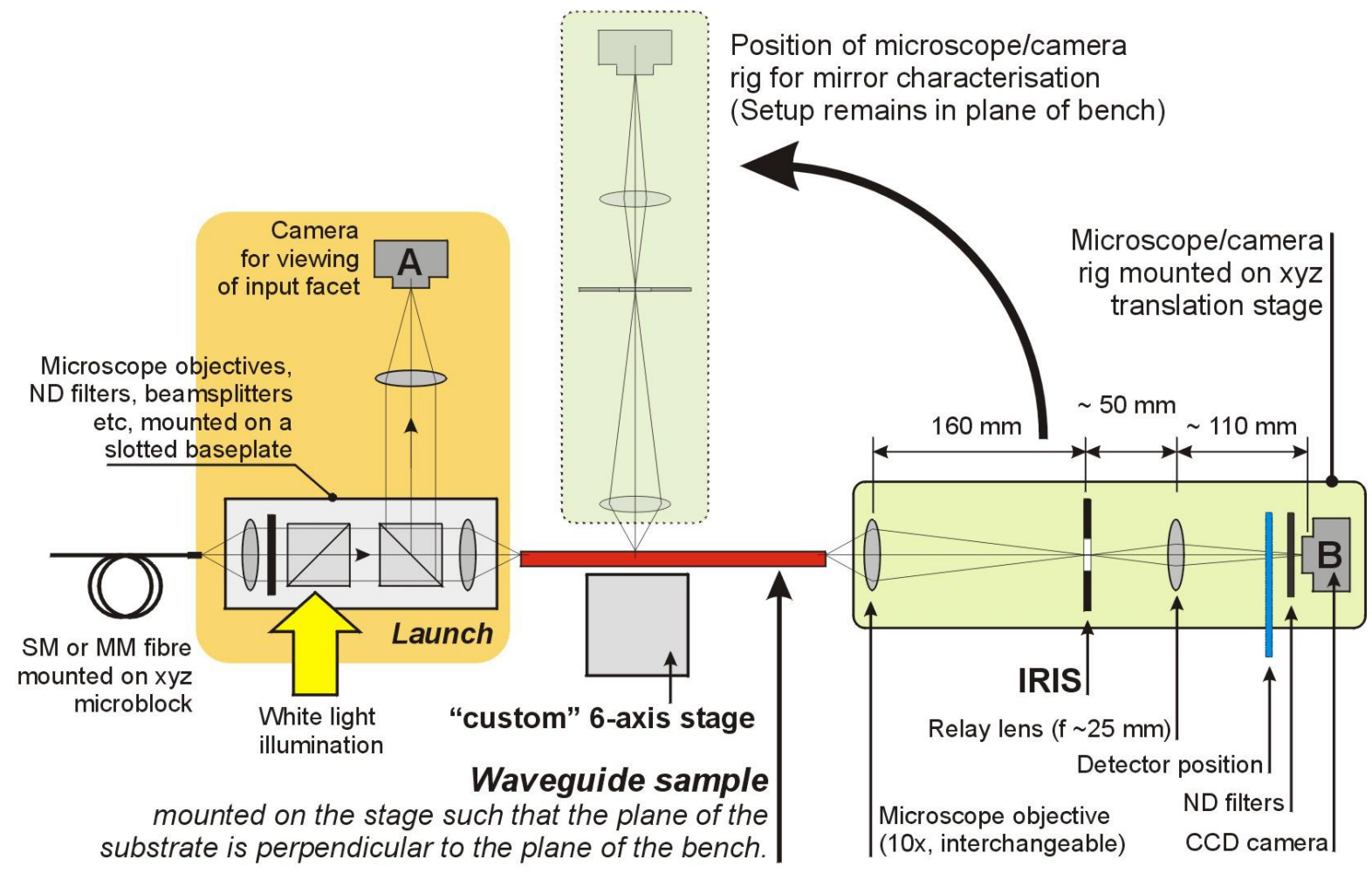

Figure 9: characterization set-up available at HWU. 


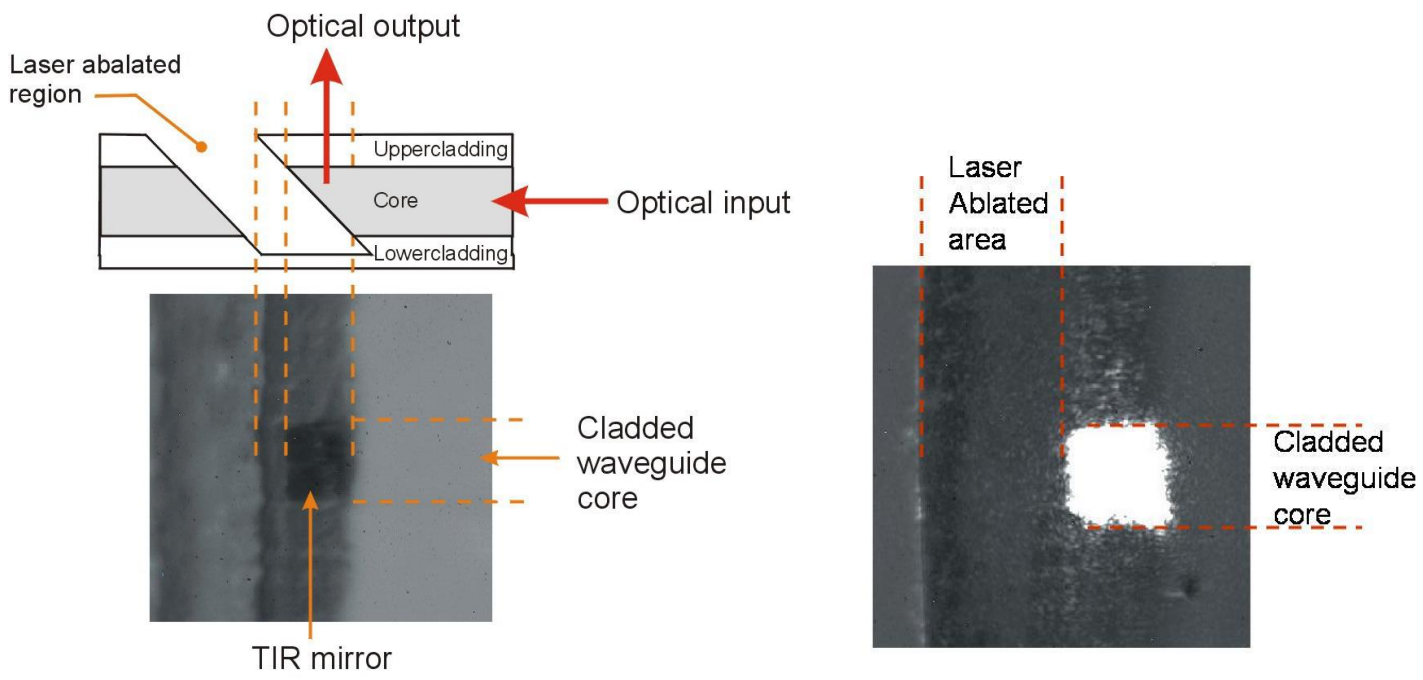

Figure 10: light spots coupled out vertically, the light is deflected over $90^{\circ}$ by the micro-mirrors out of the plane of the waveguides.

\section{CONCLUSION}

This paper reports on the use of laser direct writing and laser ablation as enabling technologies for the definition of multimode waveguides and out-of-plane turning mirrors. Surface roughness and loss measurements are performed on the waveguides and the micro-mirrors. The surface roughness of the negative facets is still to be optimized; work in this field is currently in progress. An alternative configuration, that is already being studied, is the use of a metallized positive facet. First tests show that surface roughness of these positive facets is lower than the one of the negative facets. This solution is therefore interesting and will be further investigated in the future.

\section{ACKNOWLEDGMENTS}

Nina Hendrickx would like to acknowledge the Flemish IWT (Institute for the promotion of Innovation by Science and Technology in Flanders) for financial support. This work was carried out in the framework of the NEMO-project (Network of Excellence in Micro-Optics) supported by the European Commission through the FP6 program.

\section{REFERENCES}

1. D. A. B. Miller, and H. M. Ozaktas, "Limit to the bit-rate capacity of electrical interconnects from the aspect ratio of the system architecture", Journal of parallel and distributed computing, 41, (42-52) 1997

2. K. Vandeputte, P. Van Daele, E. Hoedt, J. Van Koetsem, J. Hossfeld, "Low cost multi-fiber add/drop multiplexer demonstration system", Proc. Of the $25^{\text {th }}$ European conference on optical communication, 1, (112-113), Nice, Sept. 1999

3. J. Kobayashi, Tohru Matsuura, Y. Hida, S. Sasaki, and T. Maruno, "Fluorinated polyimide waveguides with low polarization-dependent loss and their applications to thermo-optic switches", Journal of lightwave technology, 16, (1024-1029), 1998

4. E. Griese, "A high-performance hybrid electrical-optical interconnection technology for high-speed electronic systems", IEEE Transactions on advanced packaging 24, (375-383) 2001 
5. F.Tooley, N. Suyal, F. Bresson, A. Fritze, J. Gourlay, and A. Walker, "Optically written polymers used as optical interconnects and for hybridization", Optical Materials 17, (235-241) 2001

6. G. Van Steenberge, P. Geerinck, S. Van Put, J. Van Koetsem, H. Ottevaere, D. Morlion, H. Thienpont, and P. Van Daele, "MT-Compatible Laser-Ablated Interconnections for Optical Printed Circuit Boards", Journal of Lightwave Technology 22(9), (2083-2090) 2004 